\title{
Signal-to-Noise Ratio Evaluation of Luojia 1-01 Satellite Nighttime Light Remote Sensing Camera Based on Time Sequence Images
}

\author{
Wei Wang ${ }^{1,2,3}$, Xing Zhong ${ }^{1,3, *}$, Zhiqiang $\mathrm{Su}^{3}$, Deren $\mathrm{Li}^{4}$ and Guo Zhang ${ }^{4}$ \\ 1 Changchun Institute of Optics, Fine Mechanics and Physics, Chinese Academy of Sciences, Changchun, \\ Jilin 130033, China; hitsdww@163.com; ciomper@126.com \\ 2 University of Chinese Academy of Sciences, Beijing 100049, China \\ 3 Chang Guang Satellite Technology Co., LTD, Changchun, Jilin 130102; suzhiqiang927@163.com \\ 4 State Key Laboratory of Information Engineering in Surveying, Mapping and Remote Sensing, Wuhan \\ University, Wuhan 430079, China; guozhang@whu.edu.cn; drli@whu.edu.cn \\ * Correspondence: hitsdww@163.com; Tel.: +86-180-043-19878
}

\begin{abstract}
Signal-to-noise ratio (SNR) is an important index to evaluate radiation performance and image quality of optical imaging systems under low illumination background. Under the nighttime lighting condition, the illumination of remote sensing objects is low and varies greatly, usually ranging from several lux to tens of thousands of lux. Nighttime light remote sensing imaging requires high sensitivity and large dynamic range of detectors. Luojia 1-01 is the first professional nighttime light remote sensing satellite in the world. In this paper, we took the nighttime light remote sensing camera carried on the satellite as research object, proposed an in-orbit SNR test method based on time series images to overcome the problem of low spatial resolution. We first analyzed the process of luminous flux transmission between objects and satellite and established a radiative transfer model. By combining the parameters of large relative aperture optical system and high sensitivity CMOS device, we established SNR model and specially analyzed the effect of exposure time and quantization bits on SNR. Finally we used the proposed in-orbit test method to calculate SNR of lighting images acquired by satellite. And the measured result is in good agreement with the model predicted data. Under the condition of 10lx illumination, the SNR of typical objects can reach $27.02 \mathrm{~dB}$, which is much better than the requirement of $20 \mathrm{~dB}$ for engineering application.
\end{abstract}

Keywords: signal-to-noise ratio; nighttime light imaging; time sequence images; Luojia 1-01; radiative transfer model; radiometric calibration; in-orbit test

\section{Introduction}

Signal-to-noise ratio (SNR) is a significant technical qualification for evaluating image quality and radiation performance of remote sensing camera. It is utilized to characterize the resolution of objects on the ground under different radiance, i.e. radiometric resolution. The magnitude of SNR is closely related to the parameters of image clarity and information entropy, which determines the amount of effective information that the image can provide, and ultimately affects the detection and recognition ability of the camera to objects. The higher the value of SNR, the stronger the anti-noise interference ability of remote sensing camera, and the clearer the images acquired.

With the development of high quantum efficiency and low noise focal plane arrays and the need for engineering applications, the optical remote sensing imaging technology under low illumination background, including nighttime light imaging technology, has attracted increasing attention. As early as the 1960s, the US Defense Meteorological Satellite Program launched a low-light-level imaging camera to conduct meteorological observation for strategic reconnaissance purposes [1-6]. Remote sensing imaging technology under low illumination background is of great significance in sociology and economics [7]. By imaging for targets under low illumination background, we can obtain the lighting images of the ground and extract effective information such as vehicles and roads 
[8,9]; through long-term observation and image comparison of targets under low illumination, we can judge the economic prosperity of different cities or regions [10], and even excavate the law of human activities in specific regions [11-15] and the impact of human activities on local social $[14,16]$.

In optical remote sensing imaging under low illumination background, especially in the nighttime light remote sensing, the illumination of objects is low and varies greatly, which requires high sensitivity and large dynamic range of the detector [17]. The quality of remote sensing image degrades rapidly with the attenuation of luminous flux received by the camera in the space. When the signal electron number degenerates to the same order of magnitude as the noise electron number, the remote sensing camera can hardly detect any object. Therefore, the theoretical analysis and inorbit test of SNR for nighttime light remote sensing cameras are the key to ensure the image quality and the committed step to effectively utilize the information of objects.

Luojia 1-01 satellite is the world's first professional nighttime light remote sensing satellite, manufactured by Chang Guang Satellite Technology Co., LTD. The main optical load of Luojia 1-01 satellite is nighttime light remote sensing camera with high sensitivity imaging detector and large relative aperture optical system, which can draw the global nighttime light image within 15 days. Luojia 1-01 satellite is mainly used for global nighttime light remote sensing and validation the enhancement technology of satellite navigation, providing data for macroeconomic analysis that based on nighttime light remote sensing [18].

In this paper, we took the nighttime light remote sensing camera carried on Luojia 1-01 as the research object. First, we analyzed and established the radiative transfer model between objects and satellite. Then, based on the radiation transfer model, we analyzed the generation of the signal electron number in the imaging process and derived the calculation formula of signal electron number. Meanwhile, we analyzed the main noise sources that affecting SNR and established a new noise model. Then, based on the above analysis, a SNR model suitable for nighttime light imaging was constructed by combining optical system and detector parameters, and we estimated SNR of the system using this model. Finally, the SNR model is verified by the radiation calibration data and inorbit test data, the measured values coincided with the estimated values.

\section{Materials and Methods}

\subsection{Radiative transfer model}

For remote sensing camera, under the condition of night illumination, the light emitted by light sources transmitted to the surface of the objects, and then transmitted through the atmosphere after reflecting off the objects. Then, the transmitted and part of the scattered light through the atmosphere entered into the pupil of the camera, and the optical system converges the light energy in the operation waveband to the target surface of the detector. The detector converted these collected photons into electrons with a certain conversion efficiency according to the photoelectric conversion principle, and the electrons were stored as initial data after circuit amplification and analog-to-digital conversion. And then the initial data can be developed or subsequently applied after computer image processing. Figure 1 illustrates the radiative transfer process of nighttime light imaging [19-21].

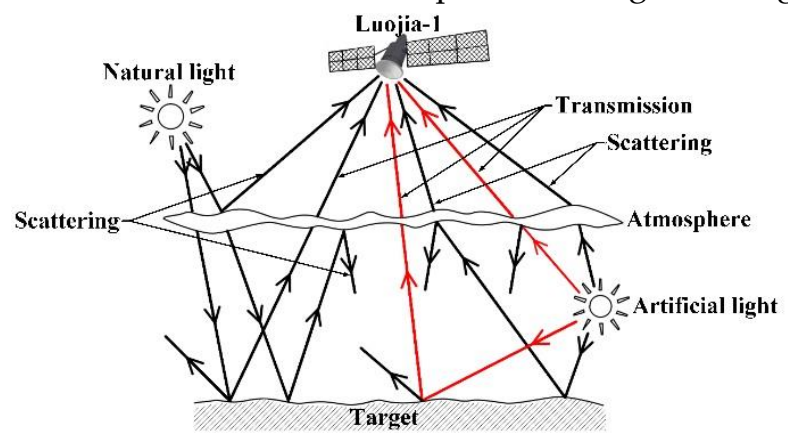

Figure 1. Radiative transfer process of nighttime light imaging.

Light sources in night include natural light sources and artificial light sources. Natural light sources include the moon and other stars, as well as light scattered by the atmosphere, however the 
most important source is the sunlight reflected from the moon and other stars or light emitted by themselves. Artificial light sources generally include all light sources produced by human activities, and the most important source is the ground lighting source. Different light sources have different brightness or illuminance on the surface of objects. Table 1 shows the brightness and illuminance of typical light sources. Although the brightness of the moon and other stars is large, the relative illumination on the ground is quite small, where the relative illumination of the moon is about $2.67 \times 10^{-1} 1 \mathrm{x}$. Even for the brightest Sirius in the night sky, that is only $1.39 \times 10^{-4} \mathrm{~lx}$ due to the long distance from the Sirius to the earth. The range of acting of artificial light sources is far less than that of natural light sources, so the illumination of artificial light sources is far greater than that of natural light sources, which are distributed in the range of 101 1031x. Therefore, only artificial light sources are considered in the radiative transfer model of nighttime light imaging.

Table 1. Typical features of different light sources.

\begin{tabular}{cccc}
\hline & Light sources & Radiance $^{\mathbf{1}}\left(\mathbf{c d} \cdot \mathbf{m}^{-2}\right)$ & Illuminance $^{\mathbf{2}} \mathbf{( 1 \mathbf { x } )}$ \\
\hline \multirow{4}{*}{ Natural } & Sirius & $1.5 \times 10^{10}$ & $1.39 \times 10^{-4}$ \\
& Moon & $2.5 \times 10^{3}$ & $2.67 \times 10^{-1}$ \\
& Night sky & $5 \times 10^{-5}$ & $2 \times 10^{-4} \sim 1 \times 10^{-2}$ \\
\hline \multirow{3}{*}{ Artificial } & Tungsten lamp & $6 \times 10^{6}$ & \\
& Automotive headlamp & $1 \times 10^{7}$ & $5 \times 10^{1} \sim 4 \times 10^{3}$ \\
& Halogen tungsten lamp & $3 \times 10^{7}$ & \\
\hline
\end{tabular}

${ }^{1}$ The radiance data are quoted from Modern Optical Engineering [22] and Engineering Optics [23].

${ }^{2}$ Besides the Sirius and the moon, the illuminance data are calculated according to the Standard for

Lighting Design of Urban Road [24] and the parameters of light sources, only the range of illuminance

is given here due to the lack of accurate data.

Artificial light sources provide illumination and are also the targets to be detected. That is, the radiance entered into the pupil of the camera includes the radiance of artificial light sources and artificial light reflected by objects. In addition, due to the scattering effect of the atmosphere, the radiance received by the pupil of the camera also includes the radiance caused by the scattering of artificial light source. The nighttime light remote sensing camera on Luojia 1-01 operates in the visible band, one of the atmospheric windows, the atmosphere has good performance of transmission in this band. Although the atmospheric transmission varies with the change in wavelength, it is kept more than $60 \%$ in the visible band. Thus in application of optical remote sensing imaging, atmospheric transmission in the visible band is usually taken as 0.6 under good atmospheric condition [25].

Atmospheric scattering can be analyzed using electromagnetic wave theory and electronic theory. When particles in the atmosphere are isotropic, the intensity of scattered light is a function of particle size, refractive index and incident wavelength. According to Bouguer's law [26], the light transmission after through the scattering of the atmosphere with a thickness of $L$ is

$$
\tau_{\beta}(\lambda, L)=\exp [-\beta(\lambda) \cdot L],
$$

where $\tau_{\beta}(\lambda, L)$ is the transmittance of scattered light, $\beta(\lambda)$ is the scattering coefficient proportional to the concentration of scattering particles. Atmospheric scattering mainly refers to Rayleigh scattering and Mie scattering $[27,28]$. The intensity of Mie scattering is two orders of magnitude lower than that of Rayleigh scattering, so here we only consider Rayleigh scattering to simplify the calculation. Rayleigh scattering is also known called molecular scattering because the particles of Rayleigh scattering are mainly gas molecules. When the radius of scattered particles is much smaller than the radiation wavelength, the atmospheric scattering obeys Rayleigh scattering rule [26,29], i.e.

$$
\beta(\lambda, \theta)=\frac{\beta(\lambda)}{4 \pi} F(\theta),
$$

where the total scattering coefficient $\beta(\lambda)$ and the phase function $F(\theta)$ can be described as 


$$
\begin{aligned}
& \beta(\lambda)=\frac{8 \pi^{2}\left(n^{2}-1\right)^{2}}{N \lambda^{4}}, \\
& F \theta=\frac{3}{4}\left(1+\cos ^{2} \theta\right) .
\end{aligned}
$$

where $n$ is the refractive index of scattering medium, $N$ is the concentration of scattering particles, and $\theta$ is the angle between scattering direction and incident direction. Under standard atmospheric pressure, the Rayleigh scattering coefficients at the wavelength $(680,550,440) \mathrm{nm}$ are $(5.8,13.5,33.1) \times 10^{-}$ ${ }^{3} \mathrm{~km}^{-1}$, the scattering transmission of these three wavelengths at the altitude of $645 \mathrm{~km}$ are $\left(2.37 \times 10^{-}\right.$ ${ }^{2}, 1.65 \times 10^{-4}, 5.346 \times 10^{-10}$ ) [29], which are much smaller than the atmospheric transmission 0.6. Combined with the data in Table 1, the illuminance of the moon and other stars is so low that the energy entered into the camera after one scattering of the atmosphere can almost be neglected. For artificial light sources, considering the multiple scattering between ground and atmosphere, even in the long wave band which is insensitive to Rayleigh scattering, the scattering transmission decreases dramatically in geometric series, and the energy received by the camera is very small.

Based on the above analysis, the radiative transfer model of the nighttime light remote sensing camera carried on Luojia 1-01satellite mainly considers the radiation that enters the pupil of the camera after atmospheric transmission, the radiation consists of artificial light sources and artificial light reflected by objects, as marked with the red solid lines in Figure 1. Since the main purpose of artificial light sources is providing ground lighting, most of the radiation are transmitted downward, so the following sections mainly analyze the artificial light reflected by objects.

\subsection{SNR model of nighttime light remote sensing camera}

\subsubsection{Signal electron number}

SNR model can be divided into energy form and photon form and we usually adopt the SNR model of photon form for imaging systems in the visible light. According to the radiative transfer model above, the signal electron number generated by a single pixel of the detector is related to the characteristics of objects, atmosphere, optical system and detector. In the process of calculating the signal electron number and evaluating SNR, the radiance entering the pupil of the camera is usually tracked and analyzed. Under low illumination conditions such as nighttime light, mirror reflection of objects occurs only in very rare cases, and in most cases it is diffuse reflection. This diffuse reflection has the characteristics of cosine radiation, i.e. Lambert radiator [22].

For Lambert radiators, the bidirectional reflectance distribution function is usually neglected [20], and the process of collecting luminous flux by a single pixel of the detector is shown in Figure 2. Figure 2(a) shows that an object with area of $A_{T}$ on the ground passes through the atmosphere with transmission of $\tau_{a}$ and be imaged by the optical system with optical transmittance of $\tau_{0}$ onto a single pixel with area of $A d$. And figure 2(b) shows that in a certain spatial angle, the radiation emitted by the object passes through the atmosphere and reaches the pupil of the camera with area of $A_{0}$, and the radiation is finally received by the detector after passing through the optical system [20].

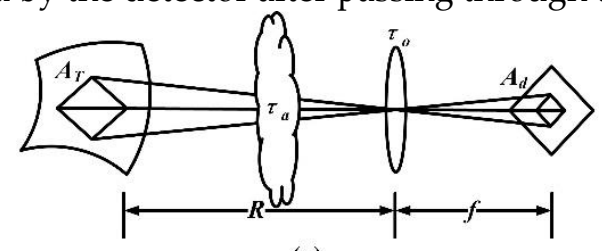

(a)

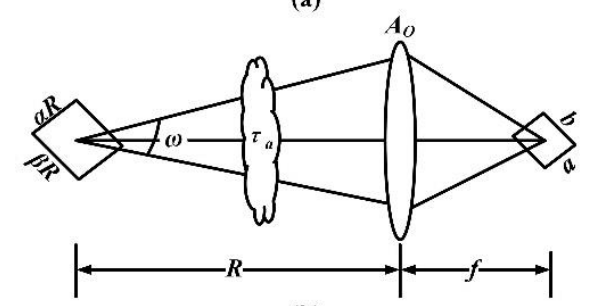

(b) 
Figure 2. (a) Imaging process from the object to the detector; (b) Radiometric transmission process from the object to the detector.

where $R$ is the distance between the camera in the space and the object on the ground, while $f$ is the focal length of the camera. $a$ and $b$ are the sizes of a single pixel, satisfying $A d=a \times b, \alpha$ and $\beta$ are the stereo angles of the object relative to the pupil of the camera, satisfying $A_{T}=\alpha R \times \beta R$. Meanwhile according to the similarity principle, the values of $\alpha$ and $\beta$ are the same as the stereo angles of a single pixel relative to the pupil of the camera, i.e. $\alpha \beta=a b / f^{2} . \omega$ is the stereo angle of the pupil of the camera with respect to the object, satisfying $\omega=A_{0} / R^{2}$.

In the spectrum of $\lambda \sim \lambda+\Delta \lambda$, assuming that the object in Figure 3 is an extended radiation source with radiance of $L \lambda$. The radiance represents the spatial distribution of radiation emitted by a radiation resource with unit area, i.e. the luminous flux emitted by the radiation source with per unit projection area and per unit stereo angle.

In Figure 3, the area of the extended source is $A_{T}$, the angle between the radiation center and the normal of the extended source is $\theta=0$, and the diameter of the pupil of the camera is $D$ whose stereo angle relative to the extended source is $\omega$. The luminous flux $\Phi_{\lambda_{0}}$ that entering the pupil of the camera after atmospheric transmission is shown as

$$
\Phi_{\lambda o}=L_{\lambda} \tau_{a}(\lambda) A_{T} \Omega \cos \theta=\frac{\alpha \beta R^{2} L_{\lambda} \tau_{a}(\lambda) A_{o}}{R^{2}}=\alpha \beta A_{o} L_{\lambda} \tau_{a}(\lambda) .
$$

After the converging of the optical system, the luminous flux $\Phi_{\lambda d}$ entering the detector is

$$
\Phi_{\lambda d}=\alpha \beta A_{o} L_{\lambda} \tau_{a}(\lambda) \tau_{o}(\lambda)
$$

The luminous flux $\Phi_{\lambda d}$ can be expressed as

$$
\Phi_{\lambda d}=\frac{a b}{f^{2}} \cdot \frac{\pi D^{2}}{4} \cdot L_{\lambda} \tau_{a}(\lambda) \tau_{o}(\lambda)=\frac{\pi A_{d} L_{\lambda} \tau_{a}(\lambda) \tau_{o}(\lambda)}{4 F^{2}} .
$$

In quantum theory, the energy of a single photon is $h v$, where $v=c / \lambda, h$ and $c$ are Planck constant and speed of light respectively. If the luminous flux on the target surface of the detector is divided by the energy of a photon, the photon number of per unit time can be obtained. According to the photoelectric conversion principle, assuming that the quantum efficiency of the detector is $\eta(\lambda)$, the signal electron number $N_{\text {signal }}$ generated by the detector in the exposure time $T_{\text {int }}$ is

$$
N_{\text {signal }}=\frac{\Phi_{\lambda d} \eta(\lambda) T_{\mathrm{int}}}{h v}=\frac{\pi A_{d} L_{\lambda} \tau_{a}(\lambda) \tau_{o}(\lambda) \eta(\lambda) T_{\mathrm{int}}}{4 F^{2} h v} .
$$

Integrating the wavelength in the operation waveband [20], Equation (6) can be expressed as

$$
N_{\text {signal }}=\frac{\pi A_{d} T_{\text {int }}}{4 F^{2} h c} \int_{\lambda} \lambda L_{\lambda} \tau_{a}(\lambda) \tau_{o}(\lambda) \eta(\lambda) d \lambda .
$$

In Equation (7), the characteristics of the radiation sources, atmospheric transmission and quantum efficiency are all functions of wavelength. If we use this precise model of integral form, we must obtain the specific forms of these parameters relating to wavelength, however that's difficult for nighttime light remote sensing imaging [30,31]. First, the spectral characteristics of different types of artificial light sources are too different to obtain a determined spectrum curve of artificial light sources. Secondly, the nighttime light remote sensing camera on Luojia 1-01 satellite acquires the gray information of objects instead of the spectral information.

To solve the problem above, we use the average value in the operating waveband to approximately replace the integral value, i.e.

$$
N_{\text {signal }}=\frac{\pi A_{d} T_{\text {int }}}{4 F^{2} h c} L \Delta \lambda \tau_{a} \tau_{o} \eta,
$$


where $L \Delta \lambda \tau_{a}$ represents the integral radiance of objects that entering the pupil of the camera. If we define $L_{e}$ and let $L_{e}=L \Delta \lambda \tau_{a}$, then the signal electron number eventually be expressed as

$$
N_{\text {signal }}=\frac{\pi A_{d} T_{\mathrm{int}}}{4 F^{2} h c} L_{e} \tau_{o} \eta .
$$

\subsubsection{Noise electron number}

The key to calculating SNR is acquiring the noise information correctly so as to separate the signal from the noise. Noise mainly occurs in the process of charge transfer and mainly caused by detectors and other electronic devices. Noise is the superposition of many factors that interfere with the signal and it can be combined with the signal through certain mathematical operations. The complete charge transfer process includes photoelectric conversion process, analog signal generation process, correlated double sampling (CDS) process and analog-to-digital conversion (A/D) process [32]. Figure 3 shows the charge transfer process [33,34] and the main noise sources, where the photogenerated current and the dark current introduce noise in the analog signal generation process, some noise can be suppressed in the CDS process, and finally the analog signal is converted into digital signal.

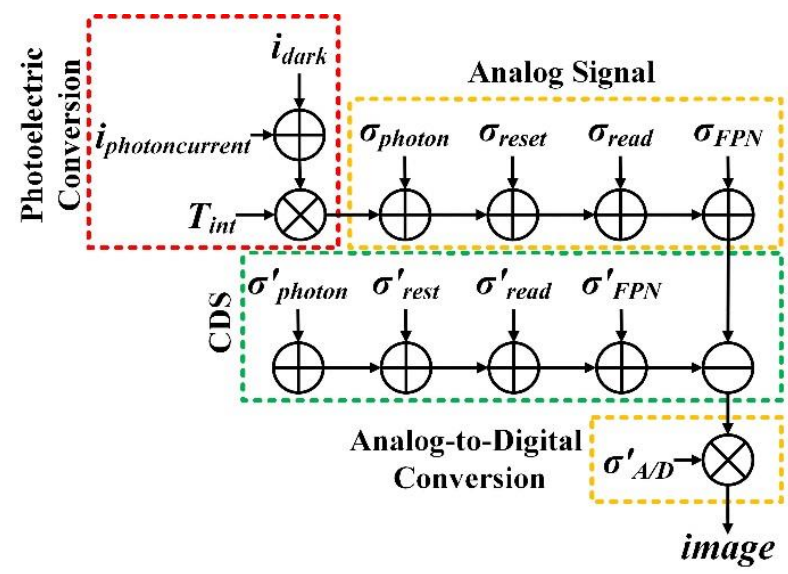

Figure 3. Charge transfer process and noise sources.

Noise of nighttime light remote sensing camera is very complex and the noise sources mainly include photon shot noise, dark current noise, fixed pattern noise, reset noise, circuit readout noise and quantization noise [35]. Previous researches show that the fixed pattern noise can be effectively suppressed or eliminated by radiometric calibration, while reset noise can be effectively suppressed of eliminated by CDS technology [36]. That is, for nighttime light remote sensing camera, the photon shot noise, dark current noise, readout noise and quantization noise are the main noise sources.

- Photon shot noise

Photon shot noise is an inherent characteristic of the camera, which is related to the characteristics of photons. The random incidence of photons results in the random generation of photo-generated electrons and consequently forms photon shot noise. The intensity of photon shot noise is proportional to the square root of incident intensity, and the SNR increases with the increase of photon shot noise. When a large number of photons incident, the photon shot noise obeys the Poisson distribution, and the standard deviation of photon shot noise is approximately equal to the square root of the signal electrons number $\sigma_{\text {photon }}=\sqrt{N_{\text {signal }}}$.

- Dark current noise

Dark current noise is random noise generated by the thermal motion of current carriers, related to the temperature of the detector closely. Due to the thermal excitation effect of the semiconductor, even in dark condition, the detector with a certain temperature can generate current due to the irregular thermal motion of electrons, making the charge number in potential wells increase, thus the dark current noise occurred. Previous research shows that adopting refrigeration measures can 
effectively suppress dark current noise. The standard deviation of dark current noise approximately equals the square root of the dark current electron number $\sigma_{\text {dark }}=\sqrt{D_{e}}$.

- Circuit readout noise

Circuit readout noise mainly refers to amplifier noise of the readout circuit, which is closely related to the circuit structure of the detector. Readout noise is additive noise, no matter how many signal incidents to a single pixel of the detector, the noise always accumulated to the signal separately. Previous research shows that readout noise can be reduced by optimizing circuit design and reducing amplifier size. The readout noise $\sigma_{\text {read }}$ can be obtained from the manual.

- Quantization noise

Analog-to-Digital conversion is a necessary process for remote sensing camera to obtain digital images. In this process, the analog signal is quantified to $2^{b}$-level digital signal, the selection of quantization bits has a significant impact on image quality [37]. Quantization noise is the difference between the signal before quantization and the signal after quantization, which is generated in $A / D$ conversion process. Quantization noise is independent of charge and related to quantization bit, reference voltage and other factors. Quantization noise cannot be completely eliminated, however, using appropriate quantization bits can reduce its impact on SNR. Under the condition of sufficient illumination, the impact of quantization noise on SNR can be neglected, while that cannot be neglected under the condition of low illumination such as nighttime light. In engineering application, the standard deviation of quantization noise is usually expressed as $\sigma_{A / D}=N_{F W} /\left(2^{b} \sqrt{12}\right)$, here the $N_{F W}$ is the full well charge of the detector [38].

Although the sources of the noise are very complex, each component of the noise is independent and non-correlated, and their effects on SNR are equivalent. According to the principle of independent error synthesis, the total noise can be expressed as the square root of the sum of squares of each noise component, and then the noise electron number of nighttime light remote sensing camera can finally be expressed as

$$
N_{\text {noise }}=\sqrt{\sigma_{\text {photon }}^{2}+\sigma_{\text {dark }}^{2}+\sigma_{\text {read }}^{2}+\sigma_{A / D}^{2}}=\sqrt{N_{\text {signal }}+D_{e}+\sigma_{\text {read }}^{2}+\frac{N_{F W}^{2}}{2^{2 b} \times 12}} .
$$

\subsubsection{Signal-to-noise ratio model}

SNR is usually defined as the ratio of signal electron number to noise electrons number $[21,39-$ 41], usually expressed in the form of decibel (dB) as follow

$$
S N R=20 \log _{10}\left(\frac{N_{\text {signal }}}{N_{\text {noise }}}\right) .
$$

We can directly obtain the illuminance data $E_{v}$ of light sources in ground test. That is, the luminous flux $\Delta \Phi$ received by unit surface of an object. Taking this object as a new light source, assuming that the reflection of the object is $\rho$, so the luminous flux emitted by unit surface of this new light source is $\rho \Delta \Phi$, which is in accordance with the definition of luminosity $M_{v}$. In this situation, the illumination and luminosity have the same physical meaning [22,42]. But the luminosity doesn't reflect the characteristics of the new light source in different directions, so it is converted into brightness $B$ in the calculation. According to the analysis in Section 2.2.1, most of the objects can be regarded as Lambert radiators, and in a space with stereo angle of $2 \pi$, the relationship between the illumination and the brightness of Lambert radiator is shown as

$$
M_{v}=\frac{d \Phi}{d A}=\pi B
$$

The brightness entering the pupil of the camera after atmospheric transmission is

$$
B=\frac{M_{v} \tau_{a}}{\pi}=\frac{E_{v} \rho \tau_{a}}{\pi} .
$$


The signal electron number in Equation (9) is deduced based on radiometry, so it is necessary to convert from photometry to radiometry. The media that connects the photometry and radiometry is spectrum power function [22], and the conversion constant for the whole space is $6831 \mathrm{~m} / \mathrm{W}$. In the space with a stereo angle of $2 \pi$, the radiance entering the pupil of the camera is

$$
L_{e}=\frac{2}{683} \cdot \frac{E_{v} \rho \tau_{a}}{\pi} .
$$

Based on the above analysis, the SNR of a single pixel is finally expressed as

$$
S N R=20 \log _{10}\left(\frac{\frac{\pi A_{d} T_{\mathrm{int}}}{4 F^{2} h c} \cdot \frac{E_{v} \rho \tau_{a}}{341.5 \pi} \cdot \tau_{o} \cdot \eta}{\sqrt{\frac{\pi A_{d} T_{\mathrm{int}}}{4 F^{2} h c} \cdot \frac{E_{g} \rho \tau_{a}}{341.5 \pi} \cdot \tau_{o} \cdot \eta+D_{e}+\sigma_{\text {read }}^{2}+\frac{N_{F W}^{2}}{2^{2 b} \times 12}}}\right),
$$

\section{Results}

\subsection{Signal-to-noise estimation of Luojia 1-01 satellite}

Luojia 1-01 satellite carries a nighttime light remote sensing camera with a large relative aperture, using domestic self-developed detector GSENSE400 with low noise, high dynamic range. Table 2 and Table 3 list some parameters of the satellite and the detector respectively.

Table 2. Parameters of Luojia 1-01 satellite.

\begin{tabular}{lc}
\hline Parameters & Values \\
\hline Operation waveband & $0.5 \sim 0.75 \mu \mathrm{m}$ \\
Central wavelength & $0.7 \mu \mathrm{m}$ \\
Optical Transmittance & $70 \%$ \\
GSD & $129 \mathrm{~m}$ \\
Relative aperture & $1: 2.8$ \\
Orbital altitude & $645 \mathrm{~km}$ \\
\hline
\end{tabular}

Table 3. Parameters of detector GSENSE400.

\begin{tabular}{lc}
\hline Parameters & Values \\
\hline Dark current $^{3}$ & $31.28 \mathrm{e}^{-} / \mathrm{s} /$ pixel $@ 25^{\circ}$ \\
Readout noise & $1.5 \mathrm{e}^{-}$ \\
Integral level & 1 \\
Full well charge & $116000 \mathrm{e}^{-}$ \\
Pixel size & $11 \times 11 \mu \mathrm{m}^{2}$ \\
Quantization bit & $15 \mathrm{bit}$ \\
\hline
\end{tabular}

${ }^{3}$ Dark current electron number is calculated as $D_{e}=31.28 \times T_{\text {int }}$.

Quantum efficiency is the proportion of the electron number produced by the detector in exposure time to the photon number that incident to the detector in exposure time. The measured quantum efficiency curve of detector GSENSE400 is shown in Figure 4. At the peak wavelength, the quantum efficiency reaches $58.21 \%$. In practical applications, we use the quantum efficiency $(\eta=0.52)$ at the central wavelength $(0.7 \mu \mathrm{m})$ as the average quantum efficiency of the whole operation band. 


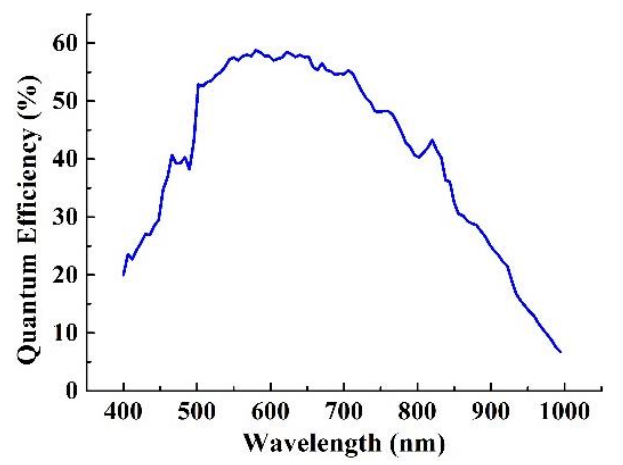

Figure 4. Quantum efficiency curve of detector GSENSE400.

\subsubsection{Effect of exposure time on SNR}

Luojia 1-01 satellite operates in push-broom imaging mode, so the exposure time is limited by velocity-to-height ratio [35], i.e. $T_{i n t} \leq G S D / V_{R}$, where $V_{R}$ is the velocity at the sub satellite point. The velocity $V_{R}$ is proportional to the in-orbit operating velocity $V_{H}$. All the velocities can be calculated by the law of universal gravitation. The calculation result indicates that in the orbit of $645 \mathrm{~km}$, the exposure time of the camera satisfying $T_{i n t} \leq 18.8686 \mathrm{~ms}$.

The longer the exposure time, the more luminous flux entering the pupil, which allows the detector to collect more effective signal, and the image quality is better [38]. Figure 5(a) shows the SNR curves under different exposure time, in which the reflection of objects on the ground is $\rho=0.3$, the atmospheric transmission is $\tau_{a}=0.6$, and the quantization bit is $b=15$. As the exposure time increased from $4 \mathrm{~ms}$ to $16 \mathrm{~ms}$, the SNR increased as a whole meanwhile. If we make the illuminance to be 1lx and keep other parameters unchanged, the curve of SNR relative to exposure time is shown in Figure 5(b). As the exposure time decreases to about $1 \mathrm{~ms}$, the SNR becomes negative, which means that the signal electron number is less than noise electron number. The image is mainly occupied by the noise. Therefore, it's necessary to prolong the exposure time appropriately for nighttime light remote sensing imaging meanwhile following the limitation of velocity-to-height ratio.
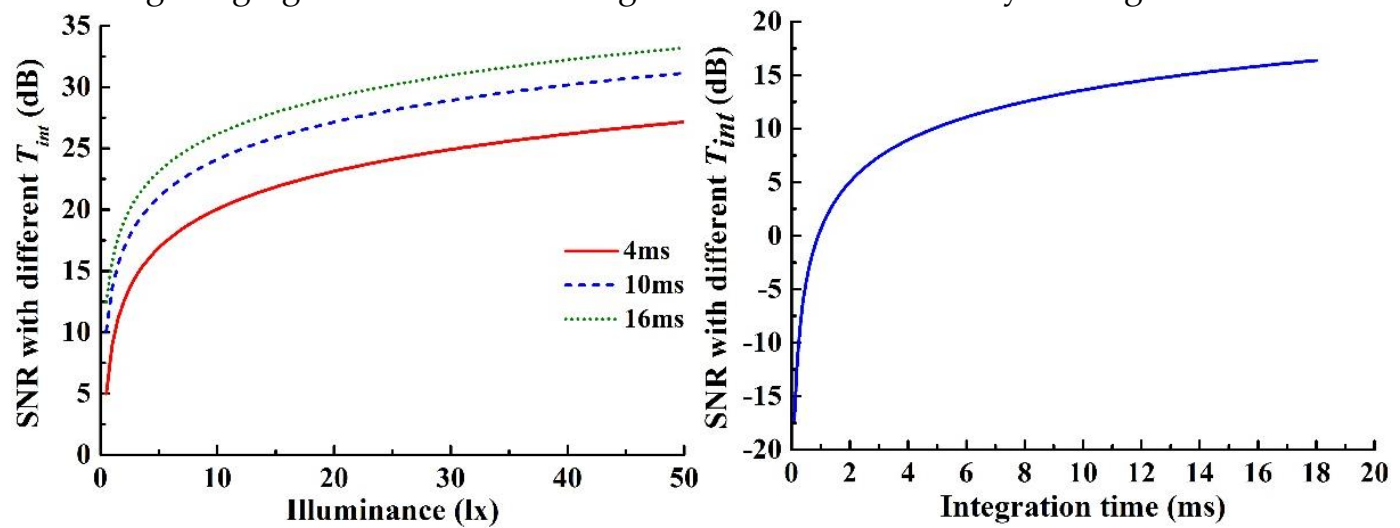

Figure 5. (a) SNR curves with different exposure time; (b) Variation curve of SNR relative to exposure time.

\subsubsection{Effect of quantization bits on SNR}

Routine remote sensing satellites usually adopt quantization bit of 10bit, while Luojia 1-01 satellite uses quantization bit of 15bit for the first time. According to the analysis in Section 2.2.2, the selection of quantization bits has a great influence on the image quality of nighttime light remote sensing camera. Figure 6(a) shows the SNR curves under different quantization bits, in which the reflection of objects on the ground is $\rho=0.3$, the atmospheric transmission is $\tau_{a}=0.6$, and the exposure time is $T_{\text {int }}=18.8686 \mathrm{~ms}$. The SNR increases as a whole with the increase of quantization bits, when the illumination is less than 5lx, the one that adopts 15bit quantization bit reaches the maximum SNR, which is more conducive to objects detection. As a comparison, those adopt 8bit and 10bit even 
appears negative SNR values. If we set the illumination to be 5lx and keep other parameters unchanged, the curve of SNR relative to quantization bit is shown in Figure 6(b). Here we set the ycoordinate to be $S / N$, i.e. ratio of signal electron number and noise electron number. SNR improves rapidly as the quantization bit increases from 8 bit to 12 bit and reaches the maximum value at 15bit quantization bit. The calculation results show that the dynamic range of Luojia 1-01 nighttime light remote sensing camera is effectively guaranteed by using 15bit quantization bit.
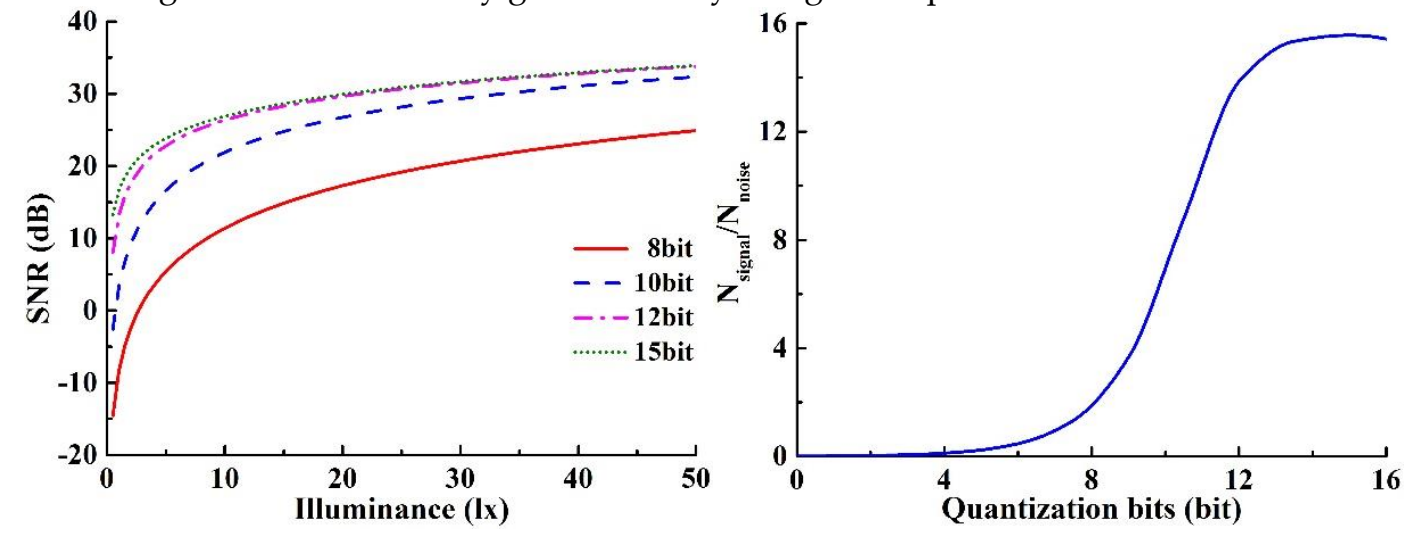

Figure 6. (a) SNR curves with different quantization bits; (b) Variation curve of SNR relative to the quantization bits.

According to engineering experience, the SNR of typical targets on the ground under 10lx illumination is about $20 \mathrm{~dB}$, and the camera fully meets the imaging standard of nighttime light imaging when the SNR reaches $20 \mathrm{~dB}$. With the help of the above SNR model and data analysis, choosing appropriate exposure time, then the SNR of Luojia 1-01 nighttime light remote sensing camera can reach at least $26.89 \mathrm{~dB}$ under $101 \mathrm{x}$ illumination.

\subsection{Signal-to-noise ratio in-orbit test}

\subsubsection{Test method}

In engineering applications, variance method is usually used to measure the SNR of remote sensing cameras. Firstly, a flat area with large area and uniform brightness distribution is selected from remote sensing images. Then calculating the average value and the deviation value of all the pixels' gray values (DN values). The proportion of the mean value to the standard deviation is defined as the SNR under a specific spectral radiance.

The relationship between the output DN value of the detector and the radiance at the pupil of the camera is usually determined by radiometric calibration $[35,43]$. Radiometric calibration is a major content in camera development, which determines the accuracy of radiometric measurement and provides support for remote sensing image correction and application [44].

Radiometric calibration device usually takes integrating sphere as light source. The uniform light emitted by the integrating sphere is collected by the camera, and the output of the integrating sphere is monitored by spectral radiometer. Changing the light intensity emitted by integrating sphere, and collecting the images under different light intensity. Through the statistical analysis of these images, we can obtain the radiometric calibration coefficients by the least square method.

The variance method cannot be used to measure the SNR of Luojia 1-01 nighttime light remote sensing camera. Luojia 1-01 satellite has a low resolution of $129 \mathrm{~m}$, which means that a target with area of $129 \times 129 \mathrm{~m}^{2}$ is projected onto a single pixel with area of $11 \mu \mathrm{m}^{2}$. Under such resolution, all the objects in the field of view of the camera are severely discretized, which means that it is difficult to select a flat area with large area and uniform brightness distribution. Therefore, variance method cannot be used for Luojia 1-01 satellite.

Variance method is essentially based on spatial sequence images [45], the pixels of the target constitute a spatial sequence, assuming the pixel number of the target is $N$. The uniform brightness 
distribution means that the outputted DN value of these $N$ pixels in this spatial sequence is approximately the same, which is equivalent to continuously acquiring $N$ images of the same target.

Based on this idea, we can convert the spatial-sequence-based method into time-sequence-based method, we extract the nighttime light remote sensing images of the same object at different time. After simple image processing, we calculate the average value and standard deviation of each pixel. And then we can obtain the SNR under different radiance in combination with the radiometric calibration coefficient. The calculation process is shown in Figure 7.

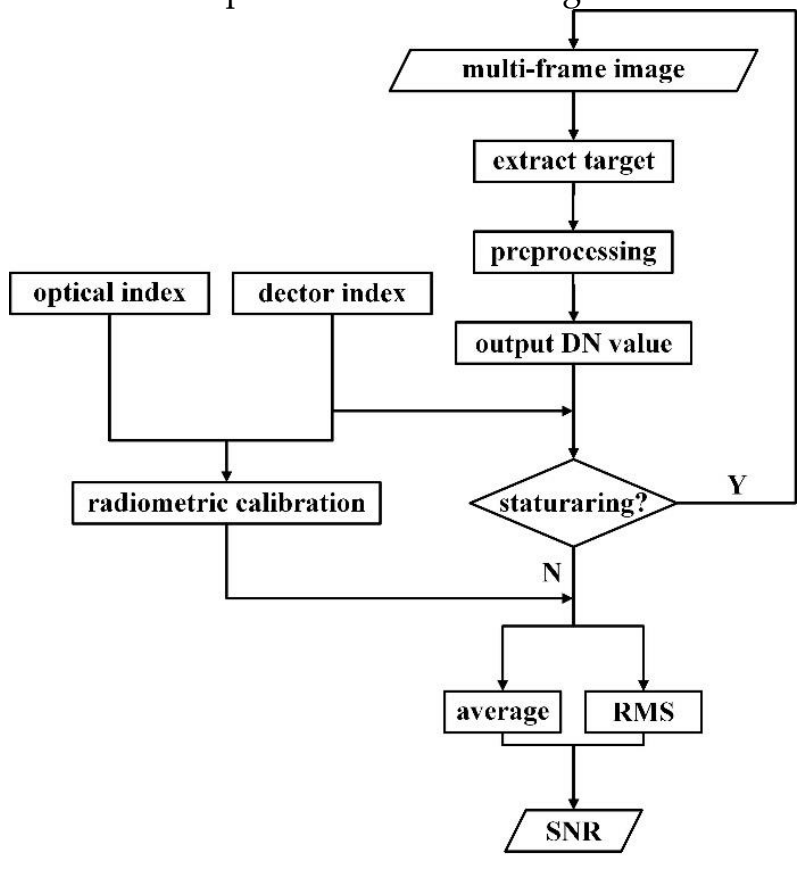

Figure 7. Calculation process of in-orbit test.

\subsubsection{In-orbit test}

Figure 8 illustrates a nighttime light image of Abu Dhabi (N242ㄱ', E54 $\left.{ }^{\circ} 3^{\prime}\right)$ captured by Luojia 1-01 satellite on June 4, 2018. The enlarged area is famous Palm Island. We extract four target areas marked with red letters from the time series images of Abu Dhabi, and the SNR of these areas is measured according to the calculation process shown in Figure 7.

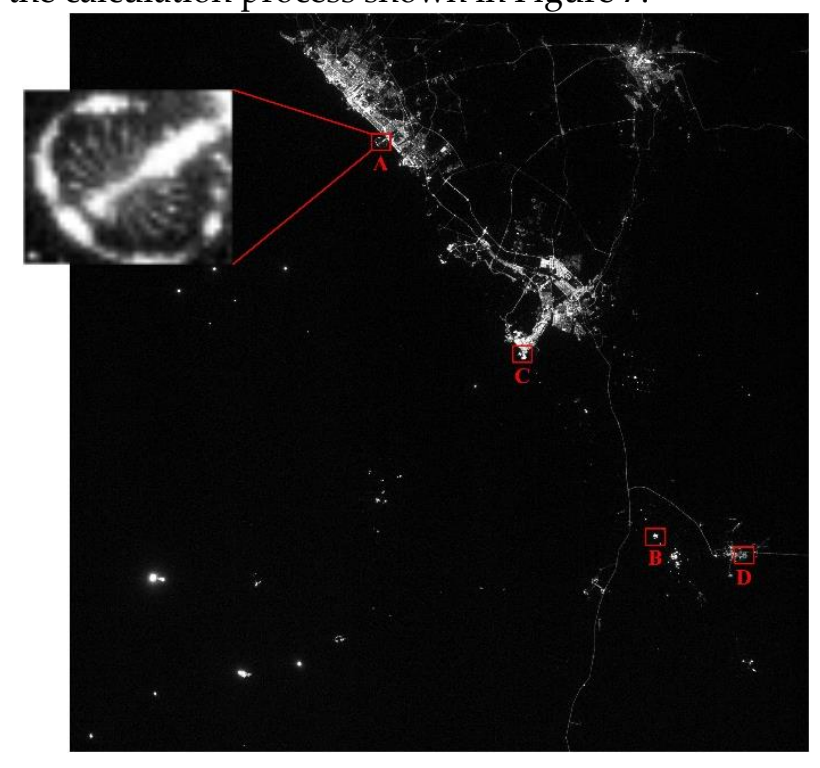

Figure 8. Nighttime light image of Abu Dhabi. 
When imaging for Abu Dhabi, the Luojia 1-01 satellite operated in push-broom mode with exposure time of $13.7 \mathrm{~ms}$ and the detector operated in HDR mode with a gain of $3.68 \mathrm{x}$. The radiometric calibration result under this situation is listed in Table 4.

Table 4. Radiometric calibration results

\begin{tabular}{ccc}
\hline Exposure time & Slope DN/(W/sr/m $\left.\mathbf{m}^{2}\right)$ & Intercept \\
\hline $2 \mathrm{~ms}$ & 3932.830 & 201.42 \\
$5 \mathrm{~ms}$ & 8797.500 & 189.43 \\
$10 \mathrm{~ms}$ & 17092.27 & 225.21 \\
$18.8 \mathrm{~ms}$ & 32913.00 & 204.48 \\
\hline
\end{tabular}

The straight line composed of Slope and Intercept represents the relationship between outputted DN value of the detector and the radiance entering the pupil of the camera. By the statistical analysis of all the data (including the data in Table 4), we found that the Slope of the response curve is linearly and positively correlated with the exposure time, while the Intercept varies near the average value. The data in $13.7 \mathrm{~ms}$ can be determined by linear fitting, which is expressed as $y=23893.58 x+205.35$, the 23893.58 and 205.35 are Slope and Intercept respectively. Combining the radiometric calibration data and the data of the optical system and detector, excluding the impact of saturation data points and dark fields, finally the statistical results are listed in Table 5.

Table 5. Statistical results of SNR in-orbit test

\begin{tabular}{cccccc}
\hline & Size & Radiance $\left(\mathbf{W} / \mathbf{s r} / \mathbf{c m}^{2}\right)$ & SNR $(\mathbf{d B})$ & Fitting & $\mathbf{R}^{\mathbf{2}}$ \\
\hline A & $33 \times 29$ & $0.88 \sim 23.58$ & $2.48 \sim 30.63$ & $y=6.991 \ln x+9.2314$ & 0.9214 \\
B & $17 \times 20$ & $0.45 \sim 13.43$ & $4.38 \sim 27.85$ & $y=4.871 \ln x+12.788$ & 0.9229 \\
C & $34 \times 25$ & $0.48 \sim 17.35$ & $4.11 \sim 30.75$ & $y=4.711 \ln x+12.452$ & 0.8816 \\
D & $16 \times 16$ & $0.50 \sim 8.420$ & $9.06 \sim 31.17$ & $y=6.572 \ln x+9.8448$ & 0.8483 \\
\hline
\end{tabular}

where Size represents the number of pixels occupied by the target area, and Fitting represents the fitting curve of SNR relative to radiance. $R^{2}$ is utilized to judge the similarity between fitting curve and real values, the larger the $\mathrm{R}^{2}$, the more accurate the fitting result. According to the statistical data in table 5, the fitting results of areas A, B and C are coincided with the measure data, and the measured data of these areas have the similar uniform distribution, which indicate that the test results are reliable in these three areas. However, the results of area D is much higher than others and the judgment coefficient is relatively small, for instance, the SNR under the illuminance of 81x is as high as $31 \mathrm{~dB}$, so these data are not reliable for reference and need to be deleted. Figure 9 displays the test results of areas $\mathrm{A}, \mathrm{B}$ and $\mathrm{C}$, where the 3D images on the left side are the distribution of SNR in spatial coordinates. The 2D images on the right side are the relation curves of SNR and radiance, where the solid blue lines are measured data, while that marked with red dots are fitting results.
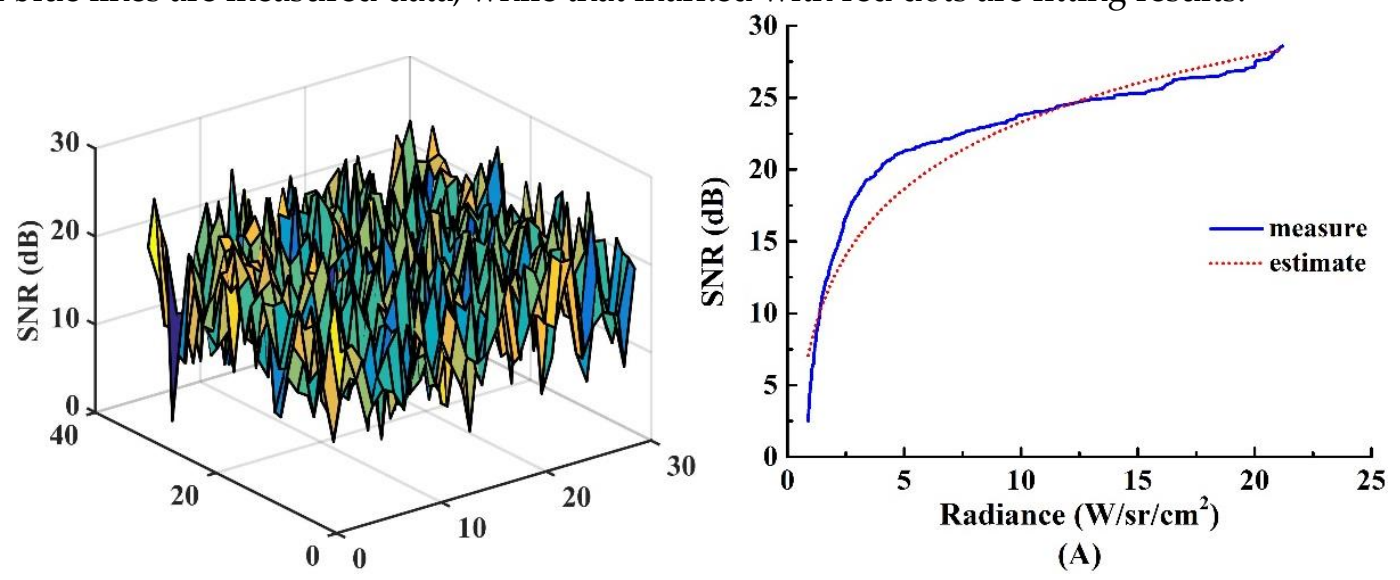

(A) 

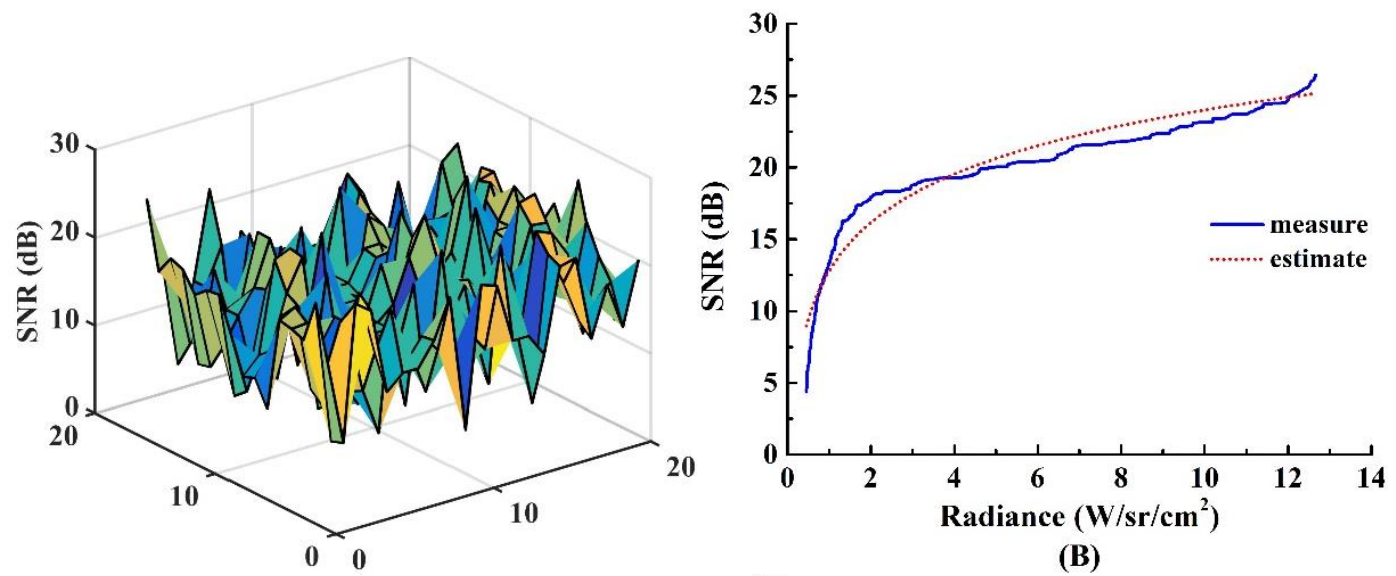

(B)
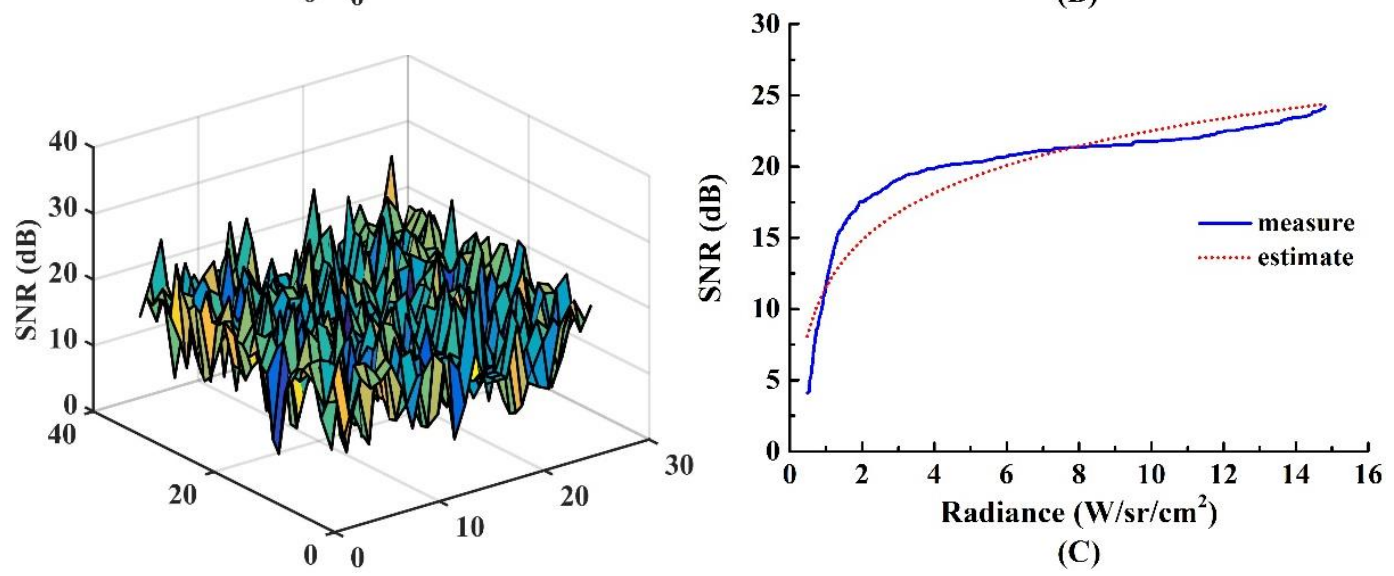

(C)

Figure 9. Images of test results of areas A, B and C.

Under the 10lx illumination at night, the SNR of typical objects usually reaches $20 \mathrm{~dB}$ before they can be applied in practical. Assuming that the reflection of the objects is 0.3 and the atmospheric transmission is 0.6, and the radiance is converted into illuminance. The test results of SNR can reach $26.29 \mathrm{~dB}, 26.73 \mathrm{~dB}$ and $27.02 \mathrm{~dB}$ at the illumination of $101 \mathrm{x}$, all higher than $20 \mathrm{~dB}$. The estimate SNR result according to the model in Section 2 under the same condition is illustrated in Figure 10 and the SNR at $101 \mathrm{l}$ is $25.49 \mathrm{~dB}$. Comparing the curves in Figure 10 and Figure 11, it can be found that the estimate data is a little small than the measured data when the radiance is lower than $10 \mathrm{~W} / \mathrm{sr} / \mathrm{cm}^{2}$, and the opposite happened when the radiance is higher than $10 \mathrm{~W} / \mathrm{sr} / \mathrm{cm}^{2}$. Generally speaking, there is a high degree of coincidence between the measured data and the estimated data, which meets the need of engineering applications.
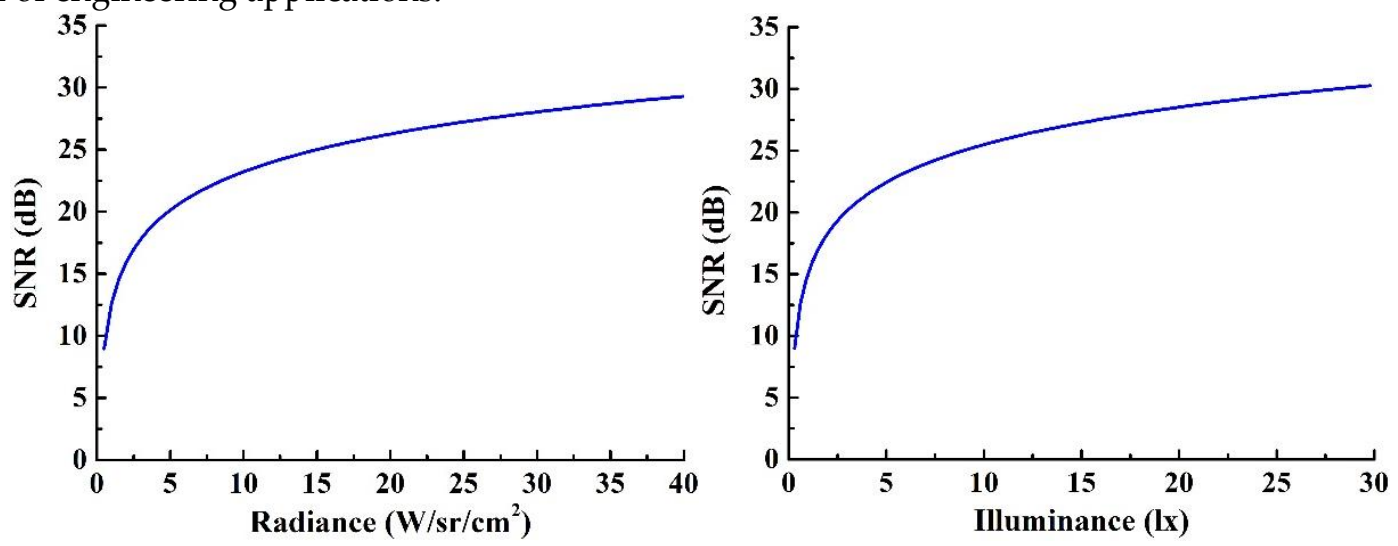

Figure 10. Estimate results of SNR in Abu Dhabi.

\section{Discussion}


Although the measured SNR data and the model predicted data of Luojia 1-01 nighttime light remote sensing camera are in good agreement, fully meets the needs of engineering applications, there are still many aspects for further discussion:

According to the analysis in Section 2, there are many factors affecting the SNR of nighttime light remote sensing camera. In this paper we only analyzed the effect of exposure time and quantization bits on SNR as shown in Figure 5 and Figure 6. The influence of reflection of objects and atmospheric transmittance on SNR also needs to be analyzed in the future.

In order to facilitate the calculation, in this paper we use the average values of each parameter to replace those parameters that are functions of wavelength, such as atmospheric transmittance, detector quantum efficiency and so on. If the parameters are refined to the magnitude of the spectrum, the SNR can better reflect the transmission of luminous flux. However, determining the accurate spectrum curve of the artificial light sources is still a hard issue to solve.

In this paper, we mainly study the SNR of the camera under nighttime light illumination, however the same camera can be used for sunlight imaging, so we can discuss the SNR of sunlight imaging. It is noteworthy that the exposure time needs to be reduced in the imaging process because of the high illuminance of the light sources during the day time.

\section{Conclusions}

In this paper, we took the first professional nighttime light remote sensing satellite, Luojia 1-01, as study object. Combined with the optical system parameters of the nighttime light remote sensing camera carried on the satellite with a large relative aperture and the parameters of rolling shutter CMOS device with large pixel and high sensitivity, we analyzed the complete luminous transmission process between the objects on the ground and the satellite in the space, and established the radiative transfer model consequently. Based on the radiative transfer model, we deduced the formulae that calculate electron signal number and noise electron number generated in the imaging process, and established the SNR model of nighttime light remote sensing camera. In push-broom imaging mode, the exposure time is limited by velocity-to-height ratio, the analysis result shows that on the premise of ensuring image quality, the exposure time must be extended reasonably in a certain range to ensure that the detector could collect enough photons. Aiming to the large variation of nighttime light illumination, Luojia 1-01 satellite firstly adopted the dynamic range extension technology with quantization bits of 15bit, which greatly improved the SNR compare with the routine remote sensing cameras. Aiming at the invalidation of traditional SNR calculation method in low-resolution images, we propose a new method that based on time series images. We conducted in-orbit SNR test of Abu Dhabi based on nighttime light images taken by Luojia 1-01 satellite using the method proposed in this paper. The results show that the measured SNR data have a high fitting linearity and are in good agreement with the predicted data of the SNR model. The measured SNR date of typical objects under the illuminance of $10 \mathrm{~lx}$ can reach $27.02 \mathrm{~dB}$, which greatly exceeds value of $20 \mathrm{~dB}$ required for engineering application. To sum up, the high sensitivity and large dynamic range ensure the ability of Luojia 1-01 satellite to detect and recognize objects in nighttime light remote sensing imaging, meanwhile the method proposed in this paper is suitable for calculating SNR of nighttime light remote sensing imaging.

Author Contributions: Writing-Original Draft Preparation and Data Curation, W.W.; Conceptualization and Methodology, X.Z.; Validation and Resources, S.Q.; Supervision, D.L. and G.Z.

Funding: This research received no external funding.

Acknowledgments: We are very grateful to Chang Guang Satellite Technology Co., LTD for their generous technical support and data providing during this research.

Conflicts of Interest: The authors declare no conflict of interest.

\section{References}

1. Elvidge, C.D.; Pettit, D.R.; Cinzano, P.; Sutton, P.C.; Small, C. In Overview of the Nightsat mission concept, Urban Remote Sensing Joint Event, 2007. 
2. Miller, S.D.; Mills, S.P.; Elvidge, C.D.; Lindsey, D.T.; Lee, T.F.; Hawkins, J.D. Suomi satellite brings to light a unique frontier of nighttime environmental sensing capabilities. P NATL ACAD SCI USA 2012, 109, 1570615711.

3. Elvidge, C.D.; Baugh, K.E.; Zhizhin, M.; Hsu, F. Why VIIRS data are superior to DMSP for mapping nighttime lights. Proceedings of the Asia-Pacific Advanced Network 2013, 35, 62-69.

4. Papitashvili, V.O.; Rich, F.J.; Heinemann, M.A.; Hairston, M.R. Parameterization of the Defense Meteorological Satellite Program ionospheric electrostatic potentials by the interplanetary magnetic field strength and direction. Journal of Geophysical Research 1999, 104(A1)-177.

5. Baugh, K.; Elvidge, C.D.; Ghosh, T.; Ziskin, D. Development of a 2009 Stable Lights Product using DMSPOLS data. Proceedings of the Asia-Pacific Advanced Network 2013, 30, 114-130.

6. Huang, Q.; Yang, X.; Gao, B.; Yang, Y.; Zhao, Y. Application of DMSP/OLS Nighttime Light Images: A Meta-Analysis and a Systematic Literature Review. REMOTE SENS-BASEL 2014, 6, 6844-6866.

7. Shi, K.; Yu, B.; Huang, Y.; Hu, Y.; Yin, B.; Chen, Z.; Chen, L.; Wu, J. Evaluating the Ability of NPP-VIIRS Nighttime Light Data to Estimate the Gross Domestic Product and the Electric Power Consumption of China at Multiple Scales: A Comparison with DMSP-OLS Data. REMOTE SENS-BASEL 2014, 6, 1705-1724.

8. Elvidge, C.D.; Safran, J.; Tuttle, B.; Sutton, P.; Cinzano, P.; Pettit, D.; Arvesen, J.; Small, C. Potential for global mapping of development via a nightsat mission. Geojournal 2007, 69, 45-53.

9. Chen, Y.L.; Chen, Y.H.; Chen, C.J.; Wu, B.F. In Nighttime Vehicle Detection for Driver Assistance and Autonomous Vehicles, International Conference on Pattern Recognition, 2006.

10. Li, X.; Xu, H.; Chen, X.; Li, C. Potential of NPP-VIIRS Nighttime Light Imagery for Modeling the Regional Economy of China. REMOTE SENS-BASEL 2013, 5, 3057-3081.

11. Franz, H.; Timothy, M.; Barbara, G.;Werner, K.;Voigt, C.C.; Dietrich, H. The Dark Side of Light: A Transdisciplinary Research Agenda for Light Pollution Policy. Ecology and Society 2010, 154(4)-art13.

12. Liu, Q.; Sutton, P.C.; Elvidge, C.D. Relationships between Nighttime Imagery and Population Density for Hong Kong. Proceedings of the Asia-Pacific Advanced Network 2011, 31, 79.

13. Jiang, W.; He, G.; Long, T.; Guo, H.; Yin, R.; Leng, W.; Liu, H.; Wang, G. Potentiality of Using Luojia 1-01 Nighttime Light Imagery to Investigate Artificial Light Pollution. SENSORS-BASEL 2018, 18, 2900.

14. Zhang, Q.; Seto, K.C. Mapping urbanization dynamics at regional and global scales using multi-temporal DMSP/OLS nighttime light data. REMOTE SENS ENVIRON 2011, 115, 2320-2329.

15. Liu, Z.; He, C.; Zhang, Q.; Huang, Q.; Yang, Y. Extracting the dynamics of urban expansion in China using DMSP-OLS nighttime light data from 1992 to 2008. LANDSCAPE URBAN PLAN 2012, 106, 62-72.

16. Li, X.; Zhao, L.; Li, D.; Xu, H. Mapping Urban Extent Using Luojia 1-01 Nighttime Light Imagery. SENSORS-BASEL 2018, 18, 3665.

17. Wang, Y.M.; Yang, X.B.; Jin, G.; Xu, W.; Zhang, Z. Study on Adaptive Exposure Time for Low-light Staring Imaging. ACTA PHOTONICA SINICA 2016, 75-81.

18. Guo, H. Luojia 1-01 Scientific Test Satellite. Satellite Application 2018, 70.

19. Thomas, G.E.; Stamnes, K. Radiative Transfer in the Atmosphere and Ocean. PHYS TODAY 2000, 53, 5758.

20. Wan, Z.; Ren, J.W.; Li, X.S.; Zhao, G.J.; Ren, J.Y. Analysis of signal-to-noise ratio for remote sensing TDI CCD camera based on radiative transfer model. Infrared and Laser Engineering 2008, 497-500.

21. Chen, Y.H.; Ji, Y.Q.; Zhou, J.K.; Chen, X.H.; Shen, W.M. Signal-to-noise ratio evaluation of airborne visibleinfrared hyperspectral imaging spectrometer. Infrared and Laser Engineering 2012, 2300-2303.

22. Smith, W.J. Modern Optical Engineering, Fourth Edition. Chemical Industry Press: Beijing, 2007, $208-223$.

23. Yu, D.Y.; Tan, Y.Y. ENGINEERING OPTICS (Fourth Edition). CHINA MACHINE PRESS: Beijing, 2006, 7780.

24. Standard for Lighting Design of Urban Road. CJJ45-20152016, Chinese Standard.

25. Zhang, Y.; Zhong, X. Signal-to-Noise Ratio in Low Light Images from Microsatellite. JOURNAL OF APPLIED SCIENCE-Electronics and Information Engineering 2017, 90-98.

26. Bai, T.Z.; Jin, W.Q. Principle and Technology of Photoelectric Imaging. BEIJING INSTITUTE OF TECHNOLOGY PRESS: Beijing 2010, 75-101.

27. Hide, R. Optics of the Atmosphere: Scattering by Molecules and Particles; 1976.

28. O'Neil, S. Accurate Atmospheric Scattering. Gpu Gems 2005.

29. Bruneton, E.; Neyret, F. Precomputed Atmospheric Scattering. COMPUT GRAPH FORUM 2008, 27, 10791086.

30. Elvidge, C.D.; Keith, D.M.; Tuttle, B.T.; Baugh, K.E. Spectral identification of lighting type and character. 
SENSORS-BASEL 2010, 10, 3961-3988.

31. Oudrari, H.; McIntire, J.; Xiong, X.; Butler, J.; Ji, Q.; Schwarting, T.; Angal, A. An Overall Assessment of JPSS-2 VIIRS Radiometric Performance Based on Pre-Launch Testing. REMOTE SENS-BASEL 2018, 10, 1921.

32. Zhang, C.; Sun, S.L.; Shi, W.X.; Wang, F.; Deng, D.X. Linear CCD camera System for industry measurement and its noise evaluation. Optics and Precision Engineering 2016, 2532-2539.

33. Jacquot, B.C.; Bolla, B.M.; Maguire, S. In Hybrid approach to mean-variance and photon transfer measurement, Image Sensing Technologies: Materials, Devices, Systems, \& Applications II, 2015.

34. Janesick, J.R. Photon Transfer DN $\rightarrow \lambda$. Photon Transfer DN $\rightarrow \lambda$. ISBN 978-0-8194-6722-5. Published by SPIE, P. O. Box 10, Bellingham, WA USA, 2007. 2007, 5249.

35. Liu, Z.X.; Wan, Z.; Li, S.X.; Li, B.Y.; Shao, Y.R. Influence factors on SNR of TDICCD space camera. Optics and Precision Engineering 2015, 1829-1837.

36. Huang, Q.L.; Jin, W.Q.; Zhu, M. TDICCD Video Data Sampling Technique for Space Remote Sensing Camera. SPACECRAFT RECOVERY \& REMOTE SENSING 2002, 23, 17-25.

37. Tan, W.; Yang, B.X.; He, H.Y. Choosing of Quantization Bits of CCD Camera for Remote Sensing Satellite. SPACECRAFT RECOVERY \& REMOTE SENSING 2014, 44-50.

38. Zhang, Y.T.; Sun, D.X.; Liu, Y.N. Study of SNR of Low Light Level Imaging System Based on CMOS Image Sensor. INFRARED 2018, 1-7.

39. Cao, Y.M.; Zhang, W.; Cong, M.Y. Signal-to-noise ratio calculation and analysis of satellite borne infrared staring camera. Journal of Astronautics 2007, 955-959.

40. Dietrich, O.; Raya, J.G.; Reeder, S.B.; Reiser, M.F.; Schoenberg, S.O. Measurement of signal-to-noise ratios in MR images: Influence of multichannel coils, parallel imaging, and reconstruction filters. JMAGN RESON IMAGING 2007, 26, 375-385.

41. Li, L.; Li, M.; Zhang, Z.; Huang, Z.L. Assessing low-light cameras with photon transfer curve method. J INNOV OPT HEAL SCI 2016, 09.

42. Zhong, X.; Jin, G.; Zhang, L. Target based SNR analysis of spaceborne panchromatic TDICCD camera. Chin, J, Space Sci 2009, 45-49.

43. Elvidge, C.D.; Baugh, K.E.; Dietz, J.B.; Bland, T.; Sutton, P.C.; Kroehl, H.W. Radiance Calibration of DMSPOLS Low-Light Imaging Data of Human Settlements. REMOTE SENS ENVIRON 1999, 68, 77-88.

44. Zhang, G.; Li, L.; Jiang, Y.; Shen, X.; Li, D. On-Orbit Relative Radiometric Calibration of the Night-Time Sensor of the LuoJia1-01 Satellite. SENSORS-BASEL 2018, 18, 4225.

45. Xu, W.; Long, X.X.; Li, Q.P. Radiometric Image Quality Assessment of GF-2 Satellite PMS Camera. SPACECRAFT RECOVERY E REMOTE SENSING 2015, 1-9. 